\title{
Visualization of multidimensional histograms using hypervolume techniques
}

\author{
Miroslav Morháč ${ }^{1}$ \\ Institute of Physics, Slovak Academy of Sciences, Bratislava, Slovakia \\ E-mail:Miroslav.Morhac@savba.sk

\section{Vladislav Matoušek and Ivan Turzo} \\ Institute of Physics, Slovak Academy of Sciences, Bratislava, Slovakia
}

\begin{abstract}
The paper presents the hypervolume visualization techniques of multidimensional nuclear spectra. The geometrical imagination of multidimensional space, its evolution from threedimensional to four-, and five-dimensional spaces is presented as well. The principles of techniques to determine the occlusion and invisibility are outlined. The paper focuses on presentation of nuclear spectra. However, the majority of algorithms can be successfully applied for visualization of other types of data as well.
\end{abstract}

XII Advanced Computing and Analysis Techniques in Physics Research Erice, Italy

3-7 November, 2008

Speaker 


\section{Introduction}

Most scalar visualization techniques use a consistent approach across one-, two-, or threedimensional fields. A scalar variable is a single quantity, in the case of nuclear spectra - counts, which can be represented as a function of independent variables - particle energies $[1,2]$. The goal of the paper is to propose a technique that allows one to localize and scan interesting parts (peaks) in the spectra of higher dimensions. Moreover it should permit to find correlations in the data, mainly among neighboring points, and thus to discover prevailing trends around multidimensional peaks.

\section{Hypervolume visualization}

This kind of visualization is designed to provide simple and fully explanatory images that give comprehensive insight into the global structure of scalar fields of any dimension [3]. Projection of m-dimensional $(\mathrm{mD})$ space to two-dimensional space can be defined as mapping

$$
\Pi\left(\mathfrak{R}^{m} \rightarrow \mathfrak{R}^{2}\right) .
$$

In what follows, we shall consider only particle scattering display mode. The position of the channel $x_{1}, \ldots, x_{\mathrm{m}}$, on the screen is given by the linear transformation

$$
\begin{aligned}
& x=t_{11} \cdot i_{1}+t_{12} \cdot i_{2}+\ldots+t_{1 m} \cdot i_{m}+t_{1, m+1} \\
& y=t_{21} \cdot i_{1}+t_{22} \cdot i_{2}+\ldots+t_{2 m} \cdot i_{m}+t_{2, m+1}
\end{aligned}
$$

or in matrix form

$$
\left[\begin{array}{c}
x \\
y
\end{array}\right]=\left[\begin{array}{lllll}
t_{11} & t_{12} & \ldots & t_{1 m} & t_{1, m+1} \\
t_{21} & t_{22} & \ldots & t_{2 m} & t_{2, m+1}
\end{array}\right]\left[\begin{array}{c}
i_{1} \\
i_{2} \\
\vdots \\
i_{m} \\
1
\end{array}\right],
$$

where

$$
\begin{aligned}
& x_{1}=x_{1, \min }+k_{1} \cdot i_{1} ; \ldots x_{m}=x_{m, \text { min }}+k_{m} \cdot i_{m} ; \\
& k_{1}=\frac{x_{1, \max }-x_{1, \min }}{n_{1}} ; \ldots k_{m}=\frac{x_{m, \max }-x_{m, \min }}{n_{m}} ;
\end{aligned}
$$

$n_{1}, \ldots n_{m}$ are the numbers of nodes of a regular grid and $X_{1}, \ldots, X_{\mathrm{m}}$ are the channels of $\mathrm{mD}$ spectrum.

The transform coefficients $t_{1} i, t_{2}{ }_{i}$ (where $\left.i=1,2, \ldots, m+1\right)$, reflect shifts, in both original and transformed space, scaling in all dimensions, as well as rotations in any direction. Shifts and scaling in $\mathrm{mD}$ space are analogous to that in conventional visualization techniques in $1 \mathrm{D}, 2 \mathrm{D}$, or $3 \mathrm{D}$ spaces. 


\subsection{Geometrical imagination of multidimensional space - evolution from three-dimensional to four-dimensional space}

We can start by adding v-axis to three-dimensional space (Fig. 4 left). By moving the cube in the fourth dimension $v$ we get the four-dimensional space (Fig. 4 middle) and by removing invisible vertices and by coloring the visible planes we get more illustrative representation of the four-dimensional space (Fig. 4 right). In 4-dimensional space v-axis is orthogonal to the axes $\mathrm{x}, \mathrm{y}$ and $\mathrm{z}$. In 2-dimensional projection its position depends on the rotation of original 4dimensional space.
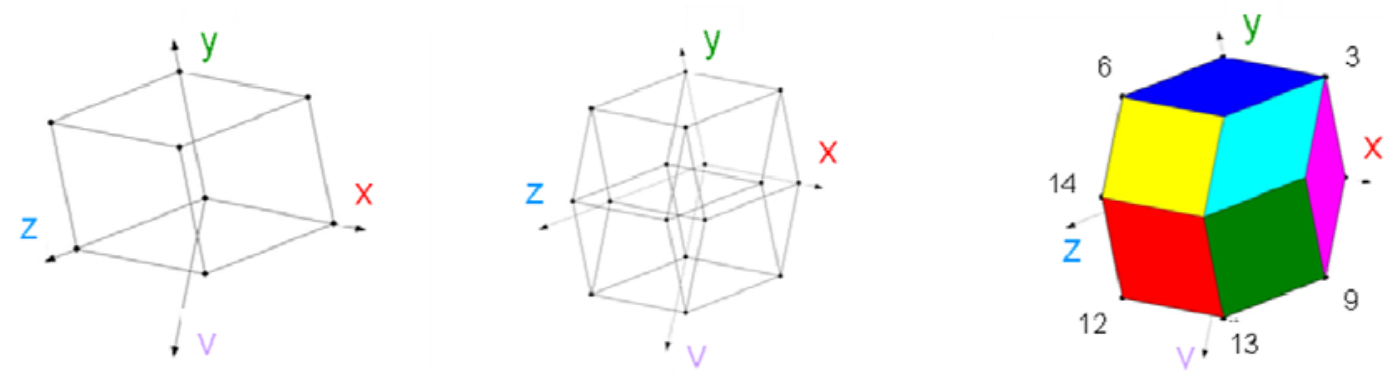

Fig. 4 Addition of $v$-axis to three-dimensional space (left), extension of three-dimensional space to four-dimensional by moving the cube in the fourth dimension (middle), and four-dimensional space (right).

\subsection{Five-dimensional space}

We can proceed in analogous way by adding w-axis to four-dimensional space and by moving the four-dimensional hypercube in the fifth dimension $w$. We get the five-dimensional space (Fig. 5).
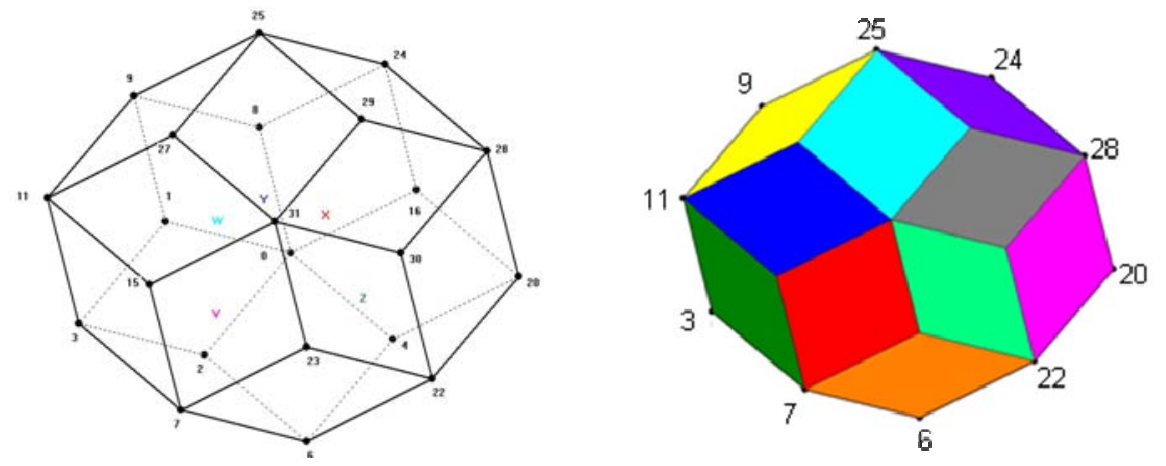

Fig. 5 Five-dimensional space with removed internal vertices and edges (left) and representation of five-dimensional space.

\subsection{Occlusion and invisibility}

In the previous sections we mentioned that some vertices and edges are "invisible". We automatically assumed the conception of real three-dimensional space. From the human point of view, this is the highest dimension we can imagine, therefore when projecting data from 3-, 4-, $5-, \ldots, m$-dimensional array we need to transform them not only to two-dimensional plane (screen) but also to three-dimensional space. Analogously to (1) the transformation to 3dimensional space is calculated 


$$
\left[\begin{array}{c}
x \\
y \\
z
\end{array}\right]=\left[\begin{array}{ccccc}
t_{11} & t_{12} & \ldots & t_{1 m} & t_{1, m+1} \\
t_{21} & t_{22} & \ldots & t_{2 m} & t_{2, m+1} \\
t_{31} & t_{32} & \ldots & t_{3 m} & t_{3, m+1}
\end{array}\right]\left[\begin{array}{c}
i_{1} \\
i_{2} \\
\vdots \\
i_{m} \\
1
\end{array}\right] .
$$

In three-dimensional space we can decide which vertices are visible and which are not. Let us define 3D space with $x y$ plane coincident with 2D space (screen) (see Fig. 6). Hence the zaxis gets automatically to be orthogonal to the screen. Then z-coordinates of the vertices give measures of distances from the observer. From them we can also determine which vertices are inside of the 3D space and which are on its surface.
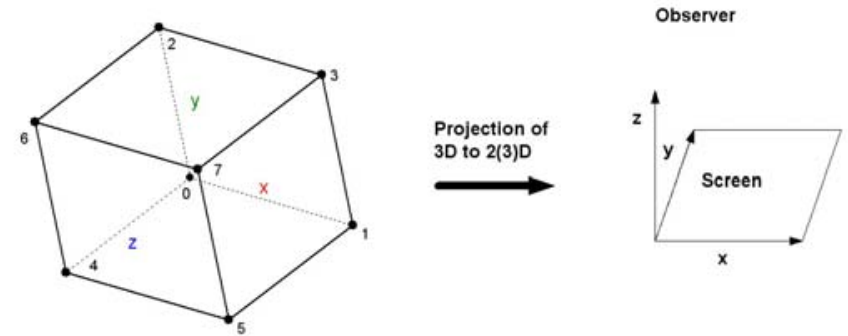

Fig. 6 Principle of the projection of $3 D$ data to $2 D$ (screen) and $3 D$ spaces.
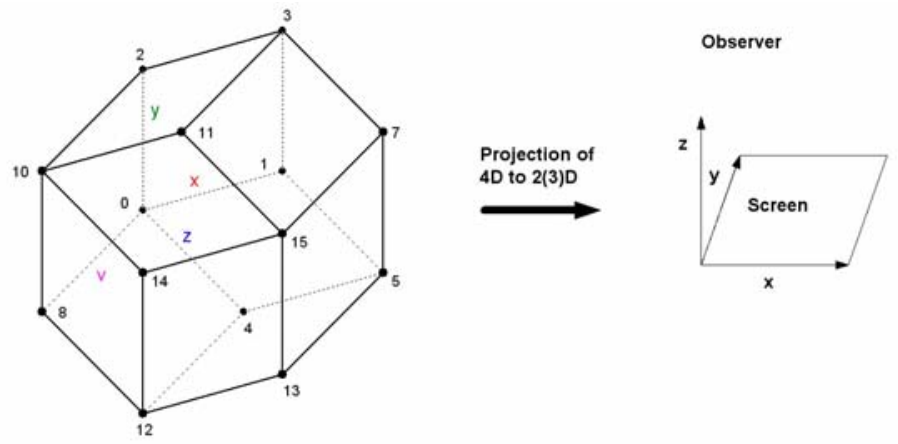

Fig. 7 Principle of the projection of $4 D$ data to $2 D$ (screen) and $3 D$ spaces.

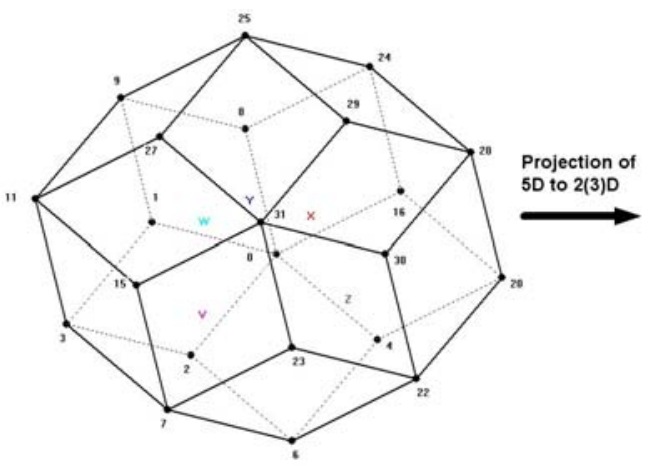

Observer

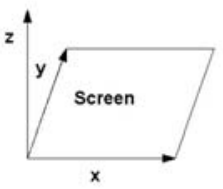

Fig. 8 Principle of the projection of $5 D$ data to $2 D$ (screen) and $3 D$ spaces.

Let us transform all vertices of $3 \mathrm{D}, 4 \mathrm{D}, 5 \mathrm{D} \ldots \mathrm{mD}$ data field to $2 \mathrm{D}$ (screen coordinate) and 3D spaces. From the z-coordinates of the transformed vertices, according to (2), we determine the backmost vertex (point), i.e., the point with the smallest value of z-coordinate (see Figs. 6, 7, 
8). It is the farthest point from the observer. It will be the starting point of the iterations in all dimensions. To the backmost vertex we determine neighboring vertices in the dimensions $x, y$, $Z, V, W, \ldots$ and we arrange them according to increasing value of z-coordinate to vector $\left[d_{1}, d_{2}\right.$, $\left.\ldots, d_{m}\right]$. Following this algorithm the points (channels) that are closer to the observer overlap the points that are farther.

\subsection{Examples of four-dimensional spectra}

To illustrate the representation of four-dimensional data we present the experimental nuclear spectrum with peaks and background in Fig. 9. The diameters of spheres represent the counts in the corresponding channels in all figures. Fig. 10 presents the synthetic fourdimensional Gaussians (left) and four three-fold slices in experimental four-dimensional $\gamma$-ray spectrum (right).
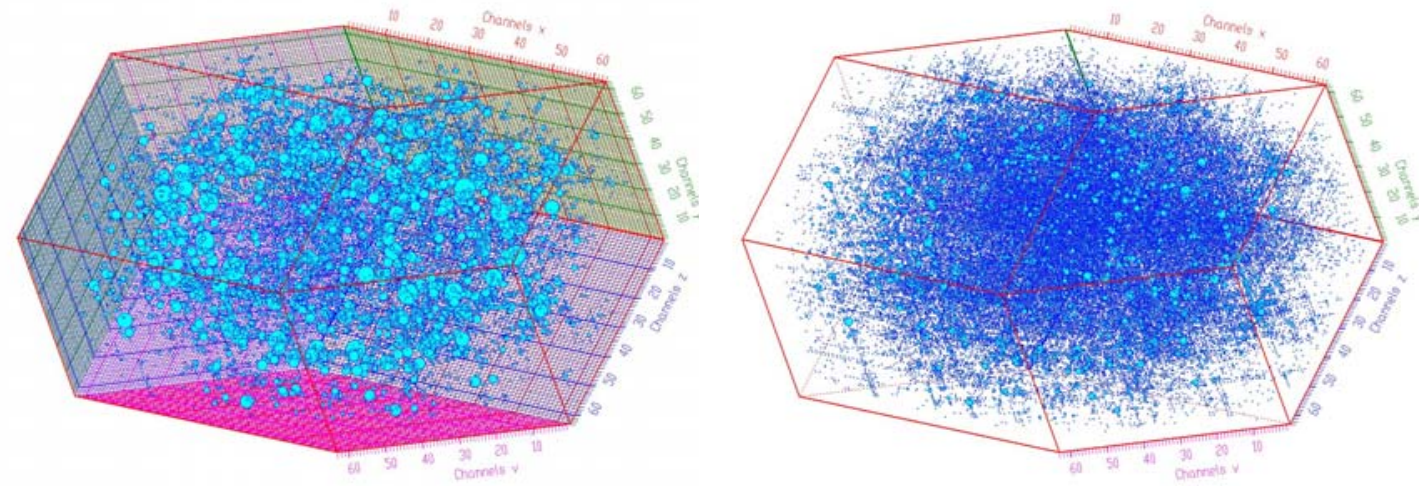

Fig. 9 Four-dimensional experimental spectrum with raster (left) and without raster (right).
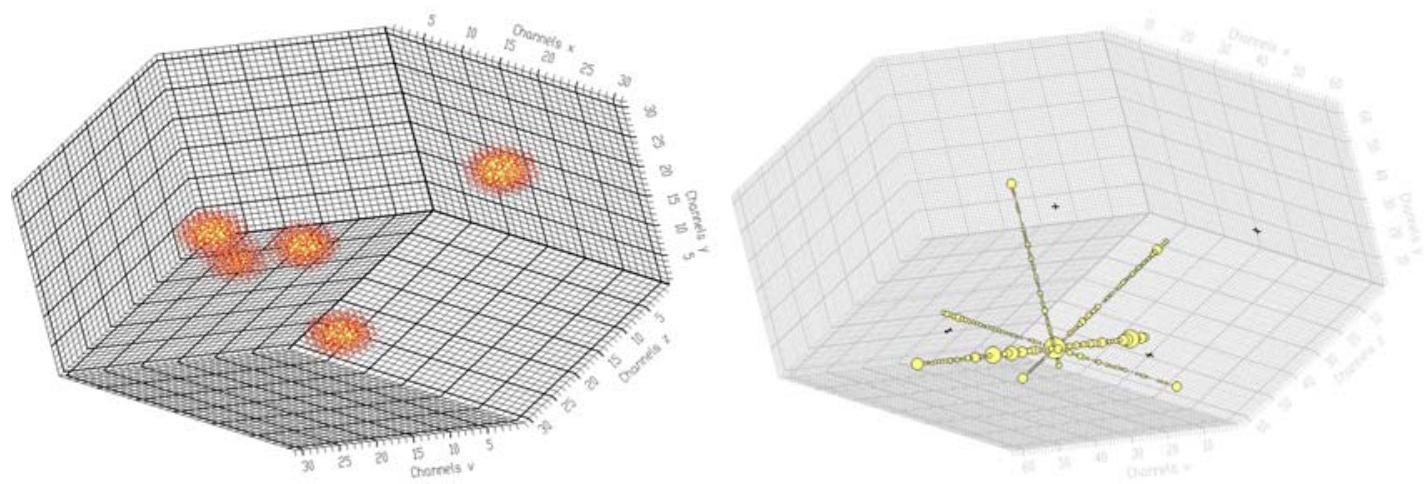

Fig. 10 Five four-dimensional Gaussians with sigma $=1.5$ (left) and four three-fold slices in experimental four-dimensional $\gamma$-ray spectrum (right).

\subsection{Examples of five-dimensional spectra}

The five-dimensional Gaussians are presented in Fig. 11. In Fig. 12 we present Gaussians with ridges (left) and 10 five-dimensional Gaussians (right). 

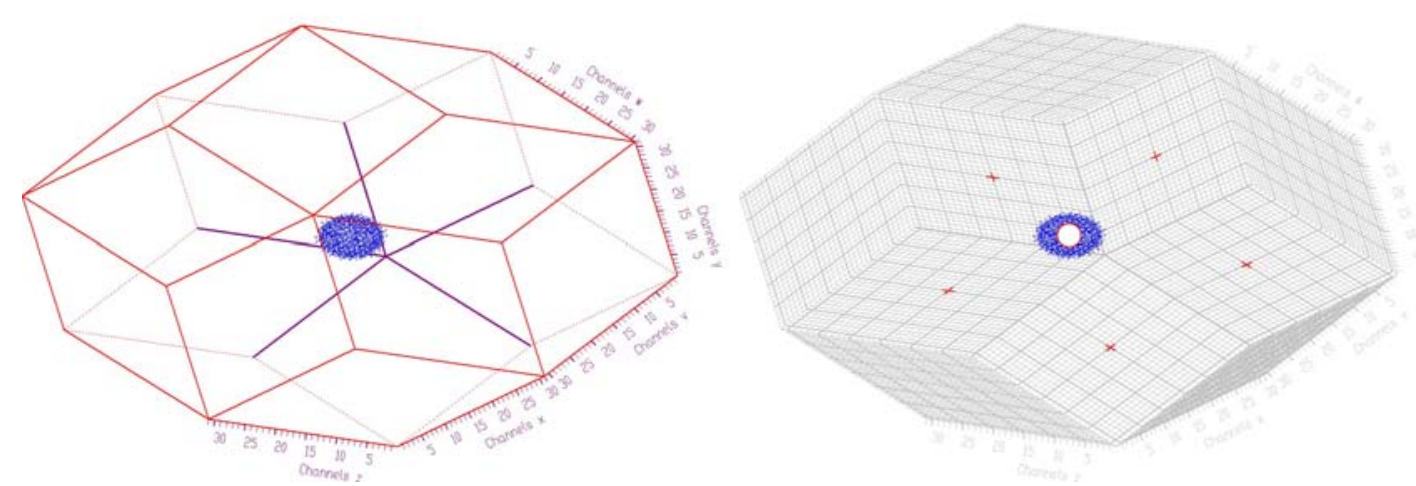

Fig. 11 Five-dimensional Gaussian. (left) and five-dimensional Gaussian with marker and raster (right).
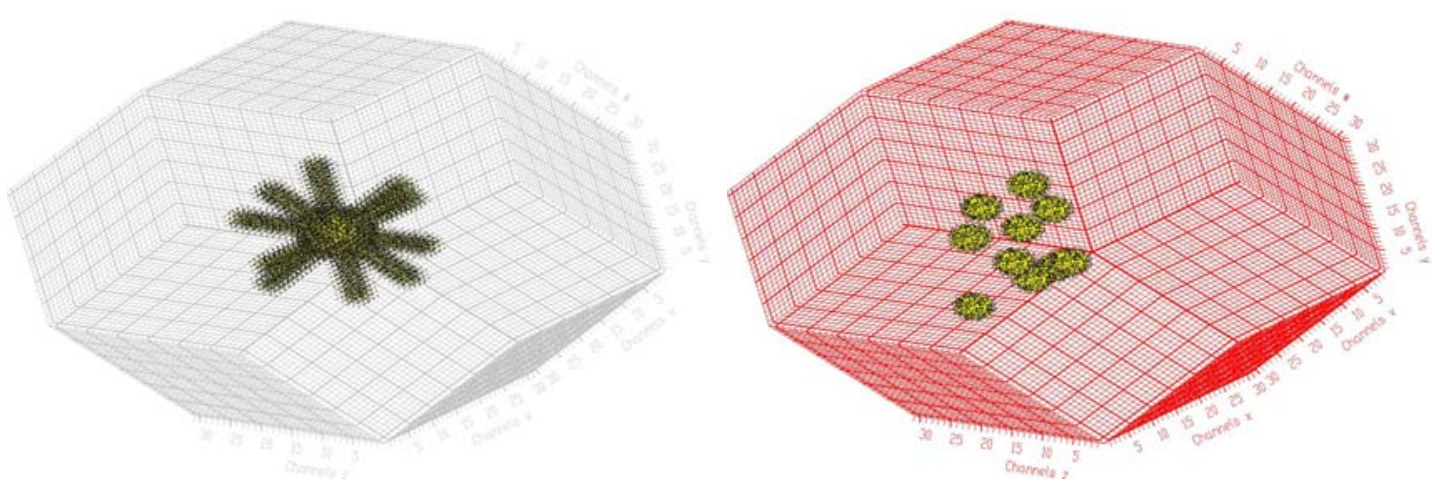

Fig. 12 Five-dimensional Gaussian with ridges (left) and 10 five-dimensional Gaussians (right).

\section{Conclusions}

Conventional volume rendering techniques are not extensible to higher dimensions. The algorithm of hypervolume visualization is presented in this contribution. It is based on particle scattering display mode. This technique is extensible to any dimension and makes it possible to provide user with compact global view of multidimensional data.

The visualization algorithms presented have been implemented in the data acquisition, processing and visualization system DaqProVis which is being developed at Institute of Physics, Slovak Academy of Sciences [4].

\section{References}

[1] Morháč M., Kliman J., Matoušek V., Turzo I., Sophisticated visualization algorithms for analysis of multidimensional experimental nuclear data, Acta Physica Slovaca 54 (2004) p. 385.

[2] Morháč M., Matoušek V.: Interactive visualization of multidimensional coincidence spectra. ACAT2007, NIKHEF, Amsterdam, Netherlands, Proc. PoS (ACAT) 064. p. 1-34, http://pos.sissa.it.

[3] C. L. Bajaj, V. Pascucci, G. Rabbiolo, D. Schikorc: Hypervolume visualization: a challenge in simplicity, Symposium on Volume Visualization, Proceedings of IEEE symposium on Volume visualization, Research Triangle Park, North Carolina, United States, pp. 95-102, 1998. 
[4] Morháč M., Matoušek V., Turzo I., Kliman J.: DaqProVis, a toolkit for acquisition, interactive analysis, processing and visualization of multidimensional data. NIM A, Vol. 559 (2006), p. 76-80. 